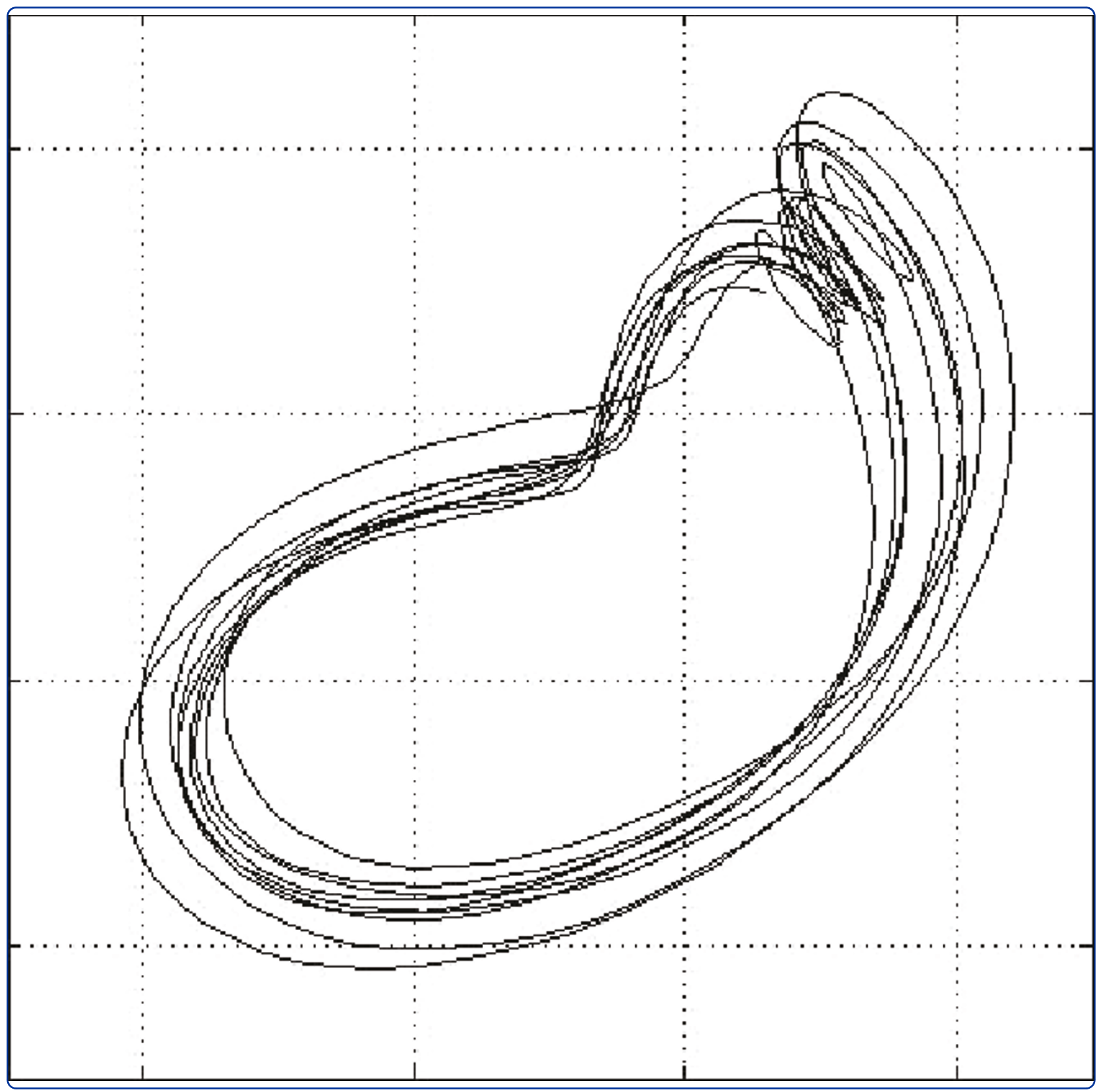

\title{
Kinematic variability, fractal dynamics and local dynamic stability of treadmill walking
}

Terrier and Dériaz 


\title{
Kinematic variability, fractal dynamics and local dynamic stability of treadmill walking
}

Philippe Terrier ${ }^{1,2^{*}}$, Olivier Dériaz ${ }^{1,2}$

\begin{abstract}
Background: Motorized treadmills are widely used in research or in clinical therapy. Small kinematics, kinetics and energetics changes induced by Treadmill Walking (TW) as compared to Overground Walking (OW) have been reported in literature. The purpose of the present study was to characterize the differences between OW and TW in terms of stride-to-stride variability. Classical (Standard Deviation, SD) and non-linear (fractal dynamics, local dynamic stability) methods were used. In addition, the correlations between the different variability indexes were analyzed.
\end{abstract}

Methods: Twenty healthy subjects performed $10 \mathrm{~min}$ TW and OW in a random sequence. A triaxial accelerometer recorded trunk accelerations. Kinematic variability was computed as the average SD (MeanSD) of acceleration patterns among standardized strides. Fractal dynamics (scaling exponent $\alpha$ ) was assessed by Detrended Fluctuation Analysis (DFA) of stride intervals. Short-term and long-term dynamic stability were estimated by computing the maximal Lyapunov exponents of acceleration signals.

Results: TW did not modify kinematic gait variability as compared to OW (multivariate $T^{2}, p=0.87$ ). Conversely, TW significantly modified fractal dynamics (t-test, $p=0.01$ ), and both short and long term local dynamic stability $\left(T^{2} p=0.0002\right)$. No relationship was observed between variability indexes with the exception of significant negative correlation between MeanSD and dynamic stability in TW ( $3 \times 6$ canonical correlation, $r=0.94)$.

Conclusions: Treadmill induced a less correlated pattern in the stride intervals and increased gait stability, but did not modify kinematic variability in healthy subjects. This could be due to changes in perceptual information induced by treadmill walking that would affect locomotor control of the gait and hence specifically alter non-linear dependencies among consecutive strides. Consequently, the type of walking (i.e. treadmill or overground) is important to consider in each protocol design.

\section{Introduction}

Walking is a repetitive movement which is characterized by a low variability [1]. This motor skill requires not only conscious neuromotor tasks but also complex automated regulation, both interacting to produce steady gait pattern. Classically, gait variability (i.a. kinematic variability) has been assessed from the differences among the strides (Standard Deviation SD, coefficient of variation $(\mathrm{CV}$ ), i.e. each stride considered as an independent event resulting from a random process. However, this approach fails to account for the presence of feedback loops in the motor control of walking: the walking pattern at a given gait cycle may have consequences on

\footnotetext{
* Correspondence: Philippe.Terrier@crr-suva.ch

${ }^{1}$ IRR, Institut de Recherche en Réadaptation, Sion, Switzerland

Full list of author information is available at the end of the article
}

subsequent strides. As a result, correlations between consecutive gait cycles and non-linear dependencies are expected.

During the last decades, various new mathematical tools have been used to better characterise the nonlinear features of gait variability. With the Detrended Fluctuation Analysis (DFA [2-4]) it has been observed that the stride interval (i.e. time to complete a gait cycle) at any time was related (in a statistical sense) to intervals at relatively remote times (persistent pattern over more than 100 strides). This dependence (memory effect) decayed in a power-law fashion, similar to scalefree, fractal-like phenomena (fractal dynamics [1,3-5]), also known as $1 / \mathrm{f}^{\beta}$ noise [6]).

Another non-linear approach was proposed to characterize the dynamic variability in continuous walking.

\section{() Biomed Central}


The sensitivity of a dynamical system to small perturbations can be quantified by the system maximal Lyapunov exponent, which characterizes the average rate of divergence in pseudo-periodic processes [7]. This method allows to evaluate the ability of locomotor system to maintain continuous motion by accommodating infinitesimally small perturbations that occur naturally during walking [8]. This includes external perturbations induced by small variations in the walking surface, as well as internal perturbations resulting from the natural noise present in the neuromuscular system [8].

Many theoretical questions are still open about the validity and application of these methods. For instance, DFA results are difficult to interpret [9], and no definitive conclusion on the presence of long range correlations should be drawn relying only on it. In addition, the underlying mechanism of long range correlations in stride interval is not fully understood $[3,10]$. West \& Latka suggested that the observed scaling in inter-stride interval data may not be due to long-term memory alone, but may, in fact, be due partly to the statistics [11]. It was also suggested that the use of multi-fractal spectrum could be a better approach than mono-fractal analysis, such as DFA $[12,13]$. There are also several methodological issues to compute consistent and reliable stability index $[14,15]$.

In parallel with the ongoing theoretical research on non-linear analysis of physiological time series, the use of non-linear bio-markers in applied clinical research has been already fruitful. In the field of human locomotion, it has been demonstrated that gait variability could serve as a sensitive and clinically relevant tool in the evaluation of mobility and the response to therapeutic interventions. For instance, gait variability (SD and dynamics) is altered in clinically relevant syndromes, such as falling and neuro-degenerative disease [16,17]. Gait instability measurement apparently predict falls in idiopathic elderly fallers [18]. Improvements in muscle function are associated with enhanced gait stability in elderly [19].

Motorized treadmills are widely used in biomechanical studies of human locomotion. They allow the documentation of a large number of successive strides under controlled environment, with a selectable steady-state locomotion speed. In the rehabilitation field, treadmill walking is used in locomotor therapy, for instance with partial body weight support in spinal cord injury or stroke rehabilitation [20,21]. Since the classical work of Van Ingen Schenau [22], it is admitted that overground and treadmill locomotion are similar if treadmill belt speed is constant. Nevertheless, both walking types present small differences in kinematics [23,24], kinetics [25] and energetics [26]. It was also observed that treadmill locomotion induced shorter step lengths and higher cadences than walking on the floor at the same speed $[26,27]$. There is still a matter of debate to interpret such subtle differences $[28,29]$.

It is obvious that treadmill walking (TW) induces specific kinaesthetic and perceptual information. Previous studies confirmed that vision plays a central role in the control of locomotion [30,31]. These differences in visual afferences between TW and Overground Walking (OW) may induce a modification in motor control, and consequently in gait variability.

In 2000, Dingwell et al. analyzed TW local dynamic stability (maximal Lyapunov exponent) in 10 healthy subjects $[8,32]$. They highlighted significant differences between TW and OW by evaluating local dynamic stability of lower limbs kinematics [8]. The effect was low in upper body accelerations. Later [32], they calculated more specifically short term stability and found a strong effect of TW in trunk accelerations. On the other hand, they found a greater kinematic variability at the lower limb level in OW as compared to TW, but no significant difference in trunk kinematics.

In 2005, Terrier et al. [1], by using high accuracy GPS, described low stride-to-stride variability of speed, step length and step duration in free walking. They observed that the constraint of rhythmical auditory signal ("metronome walking") did not alter kinematic variability, but modify the fractal dynamics (DFA) of the stride interval (anti-persistent pattern).

Based on these previous works, the working hypothesis of the present article is 1) that the constraint of TW (constant speed, narrow pathway) may induce a less persistent pattern in the stride interval, by analogy to the constraint induced by a metronome; 2) that TW may increase the local dynamic stability of walking, due to the diminution of degrees of freedom in the more constrained artificial environment [32,33], 3) that, for the same reasons, TW may slightly reduce kinematic variability [32,33] 4) that no correlation exist between the 3 variability indexes, because they are related to different aspects of the locomotion process.

The purpose of the present study was to analyze, by using trunk accelerometry, differences between TW and OW in terms of stride-to-stride kinematic variability (SD), fractal dynamics (by DFA) and local dynamic stability (maximal Lyapunov exponent). In addition, we assessed the strength of the relationships between these variables (canonical correlation analysis).

\section{Methods}

\section{Participants}

Twenty healthy male subjects, with no neurological deficit or orthopaedic impairment, participated to the study. Most of them were recruited among participants of a previous "treadmill" study implying only males subjects 
[34]. Their characteristics were (mean \pm SD): age $35 \pm$ $7 \mathrm{yr}$, body mass $79 \pm 10 \mathrm{~kg}$, and height $1.80 \pm 0.06 \mathrm{~m}$. All subjects were well trained to walk on a treadmill before the beginning of the study. The experimental protocol was approved by the local ethics committee (commission d'éthique du Valais).

\section{Apparatus}

The motion sensor (Physilog system, BioAGM, Switzerland [35]) was a triaxial accelerometer connected to a data logger recording body accelerations in medio-lateral (ML), vertical (V) and antero-posterior (AP) directions. The dimensions of the logger were $130 \times 68 \times 30 \mathrm{~mm}$ and the weight was $285 \mathrm{~g}$. The accelerometers are piezoresistive sensors coupled with amplifiers $( \pm 5 \mathrm{~g}, 500 \mathrm{mV} / \mathrm{g}$ ) and mounted on a belt. The signals were sampled at $200 \mathrm{~Hz}$ with 12-bit resolution. After each experiment, the data were downloaded to a PC computer and converted in earth acceleration units (g) according to a previous calibration. Data analysis was then performed by using Matlab (Mathworks, Natick MA, USA) and Stata 11.0 (StataCorp LP, TX, USA)

\section{Procedures}

The subjects performed $10 \mathrm{~min}$. treadmill walking (TW) and $10 \mathrm{~min}$. overground walking $(\mathrm{OW})$ in a random order. A rest period of five minutes (sitting still) was imposed between the two trials. The motor-driven treadmill was a Technogym, (Runrace, Italy). The imposed speed was $1.25 \mathrm{~m} / \mathrm{s}(4.5 \mathrm{~km} / \mathrm{h})$ for all subjects: in the context of a previous study [34], we assessed average running and walking preferred speed on the same treadmill in 88 male subjects; an average of $1.26 \pm$ $0.13 \mathrm{~m} / \mathrm{s}$ was observed. A thirty second warm-up was performed before the beginning of the measurement. For the OW test, the subjects walked along a standardized $800 \mathrm{~m}$ indoor circuit along hospital corridors and halls. The circuit exhibited only $90^{\circ}$ turns. A large part (about $400 \mathrm{~m}$ ) of the circuit was constituted by a long corridor. Other people working in the hospital were present in the halls. Hence, the OW trials mimicked actual condition of walking. Subects were asked to walk at their Preferred Walking Speed (PWS) with a regular pace. Under both conditions, the accelerometer was attached to the low back (L4-L5 region) with an elastic belt, and the logger was worn on the side of the body. Subjects wore their own low-rise comfortable walking shoes.

\section{Stride intervals and kinematic variability}

Five seconds were removed at the beginning and at the end of the $10 \mathrm{~min}$. acceleration measurements in order to avoid non-stationary periods. Heel strike was detected in the raw acceleration AP signal with a peak detection method designed to minimize the risk of false step detection: first, we generated a low-pass filtered version of the signal (4 order Butterworth, $3 \mathrm{~Hz}$, zero-phase filtering). The time of each local minimum was detected. By superimposing the Filtered Signal (FS) to the original, Unfiltered Signal (US), we tracked the nearest peak in US of each local minimum in FS. US peak time was then chosen as the limit between two steps (Figure 1A). The strides were defined as two consecutive steps. On average, the number of strides was 543 per trial.

Time series of the stride intervals were used to compute a traditional variability index (Coefficient of Variation of the stride time, $\mathrm{CV}=\mathrm{SD} /$ Mean*100, Figure $1 \mathrm{~B}$ ). Moreover, the variability of the acceleration pattern among strides was evaluated as follows (Figure 2): each stride was normalized to 200 sample points by using a polyphase filter implementation (Matlab command Resample); the average stride-to-stride Standard Deviation across all data points ((SD(i) $\left.\forall \mathrm{i} \in\left[\begin{array}{ll}1 & \ldots . .200\end{array}\right)\right)$ was evaluated $($ MeanSD $=\langle\mathrm{SD}(\mathrm{i})\rangle)$.

\section{Detrended Fluctuation Analysis}

The presence of long range correlations in the time series of stride intervals (fractal dynamics) was assessed by the use of the non-linear DFA method. Strictly speaking,
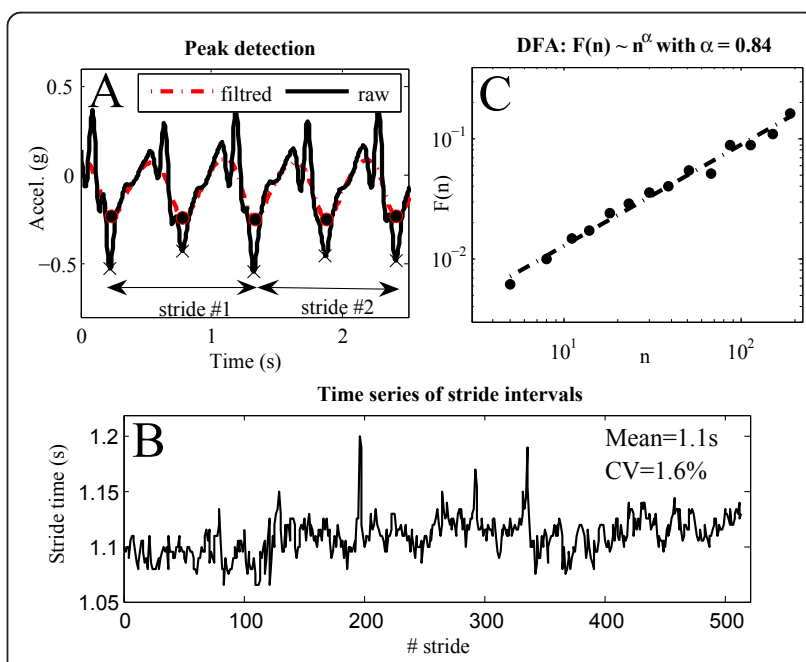

Figure 1 Method: Step detection, stride intervals and Detrended Fluctuation Analysis. One subject performed $10 \mathrm{~min}$ of free walking. A: $2.5 \mathrm{~s}$ sample of the antero-posterior acceleration signal; red dotted line is a low pass filtered $(<3 \mathrm{~Hz})$ version of the raw signal (black continuous line). Cross and black circle indicate how the algorithm specifically detect the heel strike (see method section for further explanation). The duration of two consecutive steps is defined as stride interval. B: Time series of stride intervals during the 10 min walking test. Average stride time (mean) and CV (SD/mean * 100) is also presented. C: Detredend Fluctuation Analysis (DFA). The fractal dynamics of the time series (B) is characterized by the scaling exponent $\alpha$, computed by comparing the fluctuation $(F(n))$ at different scales $(n)$ in a log-log plot. 


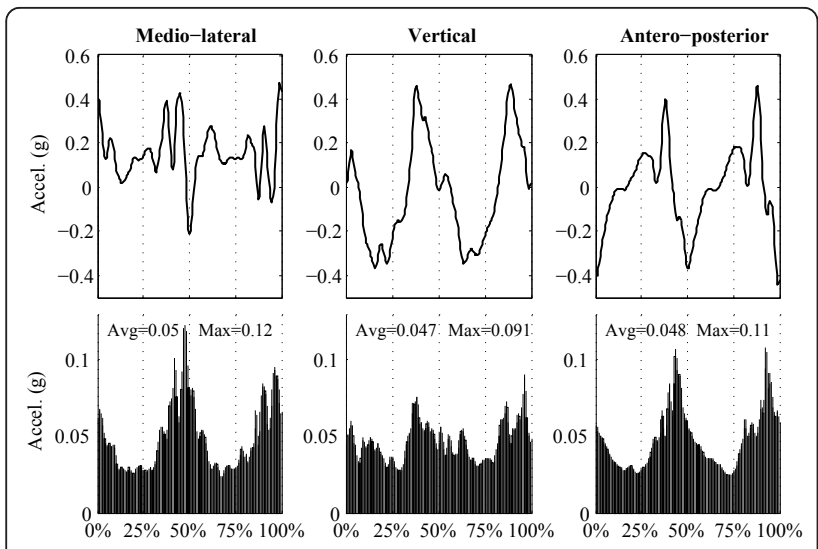

Figure 2 Method: variability, MeanSD. One subject (same as in Figure 1) performed 10 min of free walking. Each stride (see Figure 1A) was normalized to 200 samples (0\% to 100\% gait cycle). Top: Average acceleration pattern of the normalized strides $(N=513)$. Bottom: Standard Deviation (SD) of the normalized strides $(N=513)$. MeanSD is the average SD of the 200 samples.

this non-linear method should be used in addition to other statistical tools to definitively conclude that a process is a true $1 / \mathrm{f}^{\beta}$ noise with power-law decrease of long range auto-correlations [6,9]. However, DFA has been successfully used as relevant biomarker in numerous studies [1,16,17,36,37]. Detrended Fluctuation Analysis is based on a classic root-mean square analysis of a random walk, but is specifically designed to be less likely affected by nonstationarities. Full details of the methodology are published elsewhere [1-4]. In short, the integrated time series of length $N$ is divided into boxes of equal length, $n$. In each box of length $n$, a least squares line is fit to the data (representing the trend in that box). The y coordinate of the straight line segments is denoted by $\mathrm{y}_{n}(k)$. Next, the integrated time series, $\mathrm{y}(k)$, was detrended, by subtracting the local trend, $\mathrm{y}_{n}(k)$, in each box. The root-mean-square fluctuation of this integrated and detrended time series is calculated by

$$
F(n)=\sqrt{\frac{1}{N} \sum_{k=1}^{N}\left[\gamma(k)-y_{n}(k)\right]^{2}}
$$

This computation is repeated over all box sizes (from 4 to 200) to characterize the relationship between $\mathrm{F}(n)$, the average fluctuation, and the box size, $n$. The fluctuations can be characterized by the scaling exponent $\alpha$, which is the slope of the line relating $\log \mathrm{F}(n)$ to $\log (n)$ $\left(F(n) \sim n^{\alpha}\right)$, Figure $\left.1 C\right)$. Long range correlations are present in the original time series when $\alpha$ lies between 0.5 and $1[3,4]$.

In a finite length time series, an uncorrelated process could exhibit "by chance" a scaling exponent different from the theoretical 0.5 value. To statistically differentiate the stride time series from a random uncorrelated process, we applied the surrogate data method [1,3]. This method increases the confidence that the analyzed series exhibits long-range correlation. Twenty different surrogate data sets were generated by shuffling the original time series in a random order. On each data set, DFA analysis was performed to calculate $\alpha$ value. The standard deviation and mean of this sample was calculated and compared to $\alpha$ exponent of the original series. The result is considered significant if the original $\alpha$ is 2 standard deviation away from the mean of the surrogate data set.

\section{Local dynamic stability}

The method for quantifying the local dynamical stability of the gait by using largest Lyapunov exponent has been extensively described in literature [8]. It examines structural characteristics of a time series that is embedded in an appropriately constructed state space. A valid state space contains a sufficient number of independent coordinates to define the state of the system unequivocally [38]. According to the Takens' theorem, an appropriate state space can be reconstructed from a single time series using the original data and its time delayed copies (figure 3A) [38].

$$
X(t)=\left[x(t), x(t+T), x(t+2 T), \ldots, x\left(t+\left(d_{E}-1\right) T\right]\right.
$$

Where $X(t)$ is the $d_{E}$-dimensional state vector, $x(t)$ are the original data, $T$ is the time delay, and $d_{E}$ is the

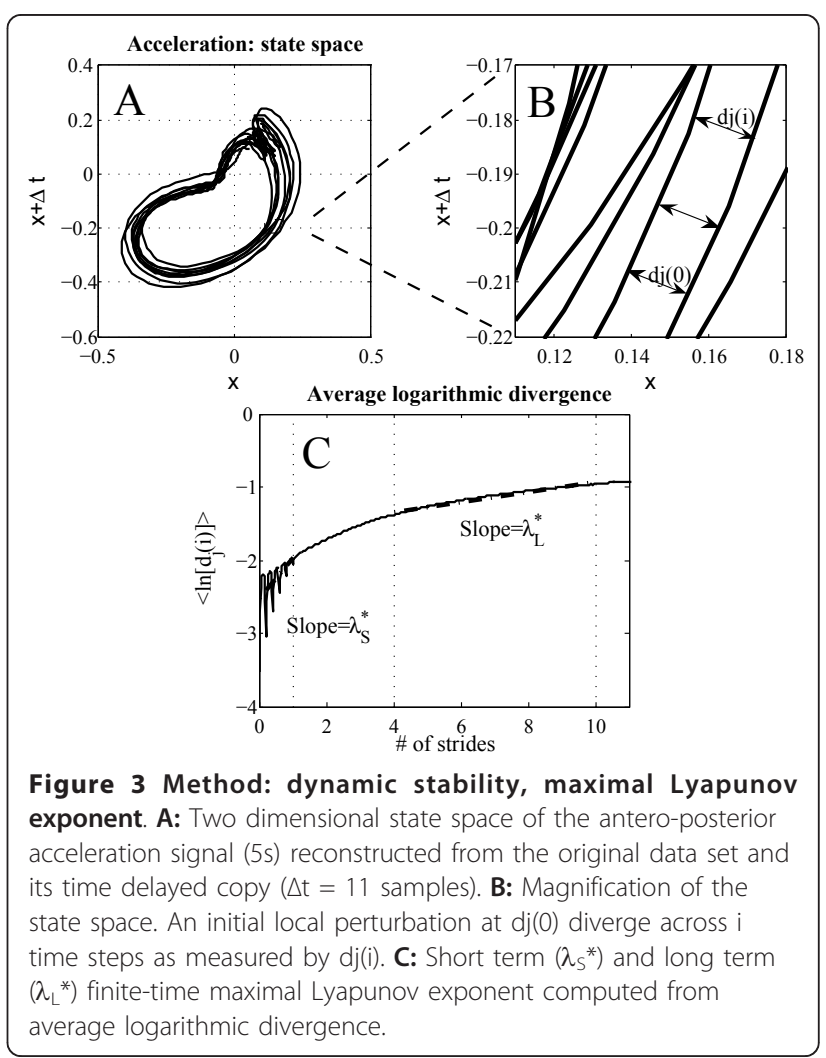


embedding dimension. The time delays $(T)$ were calculated individually for each of the 120 acceleration data set (3-axis, 2 conditions, and 20 individuals) from the first minimum of the Average Mutual Information (AMI) function [8,39]. Embedding dimensions $\left(d_{E}\right)$ were computed from a Global False Nearest Neighbors (GFNN) analysis $[8,40]$. Because the result was similar for all acceleration time series, we use a constant dimension $\left.\left(d_{E}=6\right)[8,32]\right]$. The Lyapunov exponent is the mean exponential rate of divergence of initially nearby points in the reconstructed space (Figure 3B). Because the determination of the maximal Lypunov exponent requires intensive computing power, $7 \mathrm{~min}$ of the 10 min walking test (from 1.5 to $8.5 \mathrm{~min}$.) was selected and the raw data were down-sampled to $100 \mathrm{~Hz}$. The determination of the Lyapunov exponent was then achieved by using the algorithm introduced by Rosenstein and colleagues [7], which provided dedicated software to compute divergence as a function of time in finite-time series [41] (Figure 3B). The maximum finitetime Lyapunov exponents $(\lambda *)$ were estimated from the slopes of linear fits in the divergence diagrams (Figure 3C). Strictly speaking, because divergence diagrams (Figure $3 \mathrm{C}$ ) are non-linear, multiple slopes could be defined and so no true single maximum Lyapunov exponent exists. The slopes (exponents) quantify local divergence (and hence stability) of the observed dynamics at different time scale, and should not be interpreted as a classical maximal Lyapunov exponent in chaos theory.

Since each subject exhibited a different average step frequency, the time was normalized by average stride time for each subject and each condition (Figure 3C). As suggested by Dingwell and colleagues [32], we use two different time scales for assessing short-term and long-term dynamic stability: short term exponents $\left(\lambda_{\mathrm{S}}{ }^{*}\right)$ was computed over the first stride ( 0 to 1 ), and long term exponents $\left(\lambda_{\mathrm{L}}{ }^{*}\right)$ between 4 and 10 strides (Figure 3C).

\section{Statistical analysis}

Mean and Standard Deviation (SD) were computed to describe the data (table 1). Ninety-five percent Confidence Intervals $(\mathrm{CI})$ were calculated as \pm 1.96 times the Standard Error of the Mean (SEM, N = 20).

The effect size of TW as compared to OW was expressed in both absolute (mean difference) and standardized (mean difference divided by SD) terms. The standardized effect size was the Hedge's g, which is a modified version of the Cohen's $d$ for inferential measure [42]. Paired t-tests between OW and TW were performed, and the p-values are shown in the last column of table 1. The precision of the effect sizes was estimated with CI (Figure 4). CI were \pm 1.96 times the asymptotic estimates of the standard error (SE) of $g$ [42]. The arbitrary limit of 0.5 was uses to delineate small effect size, as defined by Cohen [42]. The extent of the data (quartiles and median) and individual differences between conditions are shown in Figure 5 for $\lambda^{*}$. In order to facilitate results interpretation by reducing the risk of type I statistical error, a Hotelling $\mathrm{T}^{2}$ test was used. This is a multivariate generalization of paired t-test [43]. The null hypothesis is that a vector of $\mathrm{p}$ differences is equal to a vector of zeros. Two multivariate sets were tested: meanSD $(p=3)$ and $\lambda^{*}(p=6)$.

Canonical correlation analyses (CCA, table 2 \& 3) were performed in order to assess the strength of the

Table 1 Comparison between Overground and Treadmill Walking

\begin{tabular}{|c|c|c|c|c|c|c|c|c|c|}
\hline \multirow[b]{2}{*}{$N=20$} & & \multicolumn{2}{|c|}{ Overground Walking } & \multicolumn{2}{|c|}{ Treadmill Walking } & \multicolumn{2}{|c|}{ Effect Size } & \multirow{2}{*}{$\begin{array}{r}\text { T-test } \\
\mathbf{p}\end{array}$} & \multirow{2}{*}{$\mathrm{T}^{2}$-test } \\
\hline & & Mean $\pm S D$ & Confidence interval & Mean \pm SD & Confidence interval & Abs. & Norm. & & \\
\hline \multirow{3}{*}{ Mean variability $(S D, g)$} & $M L$ & $0.08 \pm 0.03$ & $0.07-0.09$ & $0.07 \pm 0.03$ & $0.06-0.09$ & 0.00 & -0.12 & 0.59 & \\
\hline & V & $0.08 \pm 0.03$ & $0.07-0.09$ & $0.08 \pm 0.03$ & $0.06-0.09$ & -0.01 & -0.16 & 0.48 & 0.87 \\
\hline & $\mathrm{AP}$ & $0.08 \pm 0.03$ & $0.07-0.09$ & $0.08 \pm 0.03$ & $0.07-0.09$ & 0.00 & -0.01 & 0.96 & \\
\hline \multicolumn{2}{|c|}{ Stride time (mean, s) } & $1.06 \pm 0.06$ & $1.04-1.09$ & $1.10 \pm 0.07$ & $1.07-1.13$ & 0.03 & 0.53 & 0.01 & \\
\hline \multirow{2}{*}{\multicolumn{2}{|c|}{$\begin{array}{r}\text { Stride time variability (CV, \%) } \\
\text { Scaling exponent } \alpha \text { (DFA) }\end{array}$}} & $2.74 \pm 0.87$ & $2.36-3.12$ & $3.03 \pm 1.44$ & $2.40-3.66$ & 0.29 & 0.24 & 0.43 & \\
\hline & & $0.81 \pm 0.09$ & $0.78-0.85$ & $0.72 \pm 0.13$ & $0.67-0.78$ & -0.09 & -0.80 & 0.01 & \\
\hline \multirow{3}{*}{ Short term stability $\left(\lambda^{*} \mathrm{~s}\right)$} & $M L$ & $0.75 \pm 0.11$ & $0.70-0.79$ & $0.68 \pm 0.15$ & $0.61-0.74$ & -0.07 & -0.53 & 0.01 & \\
\hline & V & $0.75 \pm 0.14$ & $0.69-0.82$ & $0.68 \pm 0.16$ & $0.61-0.75$ & -0.07 & -0.48 & 0.01 & \\
\hline & $\mathrm{AP}$ & $0.72 \pm 0.10$ & $0.68-0.76$ & $0.66 \pm 0.13$ & $0.60-0.71$ & -0.06 & -0.57 & 0.02 & 0.00 \\
\hline \multirow{3}{*}{ Long term stability $\left(\lambda_{\llcorner}^{*}\right)$} & $\mathrm{ML}$ & $0.022 \pm 0.007$ & $0.019-0.025$ & $0.018 \pm 0.008$ & $0.015-0.021$ & -0.004 & -0.60 & 0.02 & \\
\hline & V & $0.048 \pm 0.014$ & $0.042-0.054$ & $0.040 \pm 0.015$ & $0.034-0.046$ & -0.008 & -0.54 & 0.00 & \\
\hline & AP & $0.041 \pm 0.008$ & $0.038-0.044$ & $0.039 \pm 0.013$ & $0.033-0.045$ & -0.002 & -0.15 & 0.48 & \\
\hline
\end{tabular}

The Descriptive statistics of variability indexes are expressed as mean, Standard Deviation (SD) and $95 \%$ Confidence Interval (mean \pm 1.96 times the Standard Error of the Mean). The effect size is given as Absolute (Abs.) and Normalized (Norm.) values, i.e. respectively the difference between Overground (OW) and Treadmill (TW) conditions (Abs.) and the difference normalized by SD (Hedge's g). The t-test column shows the $p$ values of paired t-tests between TW and OW conditions. $T^{2}$-test is the Hotelling multivariate test by regrouping MeanSD and $\lambda^{*}$. Significant results $(p<0.05)$ are printed in bold. ML, $V$ and AP stand for respectively Medio-Lateral, Vertical and Antero-posterior, i.e. the 3 directions of the triaxial accelerometer. 


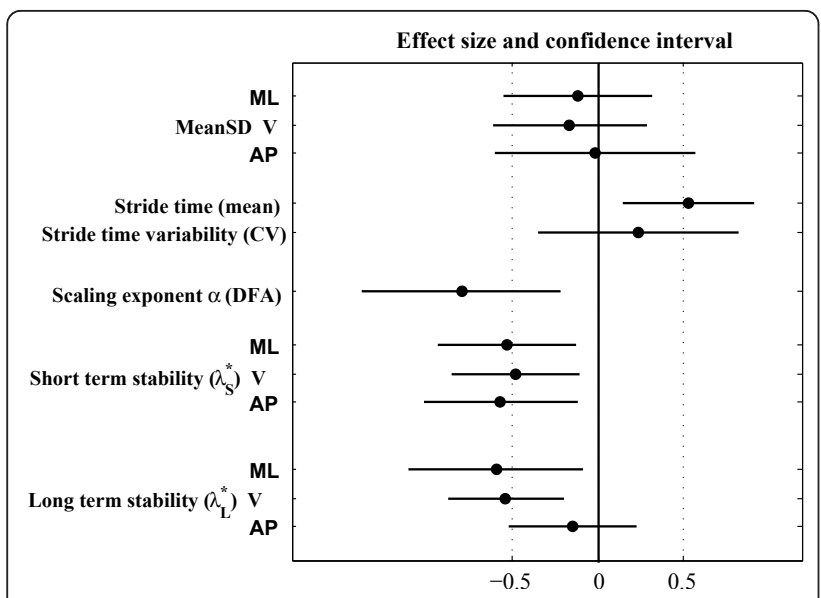

Figure 4 Differences between overground and treadmill walking. Effect size and confidence intervals. Black circles are the standardized effect size (Hedge's g), as reported in table 1. Horizontal lines are the 95\% confidence intervals. The arbitrary limit of 0.5 (vertical dotted line) corresponds to a medium effect as defined by Cohen. relationships between different sets of variables [43] This multivariate method allows one to find linear combinations (variates) in two sets of variables, which have maximum correlation (canonical correlation coefficient or canonical root) with each other. For each condition (OW and TW), two sets of $\mathrm{p}$ variables were analyzed: kinematic variability (set\#1, $\mathrm{p}=3$ ) including MeanSD in ML, V and AP directions, and dynamic stability (set\#2, $\mathrm{p}=6$ ), including short term and long term lyapunov exponent $\left(\lambda_{\mathrm{S}^{*}}, \lambda_{\mathrm{L}}{ }^{*}\right)$ in $\mathrm{ML}, \mathrm{V}$ and AP directions. In addition, $\alpha$ scaling exponent was also analyzed with the same method vs. set\#1 and set\#2. In this case, CCA is equivalent to multiple regression analysis. Significance of the canonical correlations was assessed with the Wilks' lambda statistics.

To enhance the interpretation of CCA, different parameters were computed: the standardized canonical weights are the linear coefficients for each set after Ztransform of the variables; canonical loadings are the correlation coefficients between each variable and their

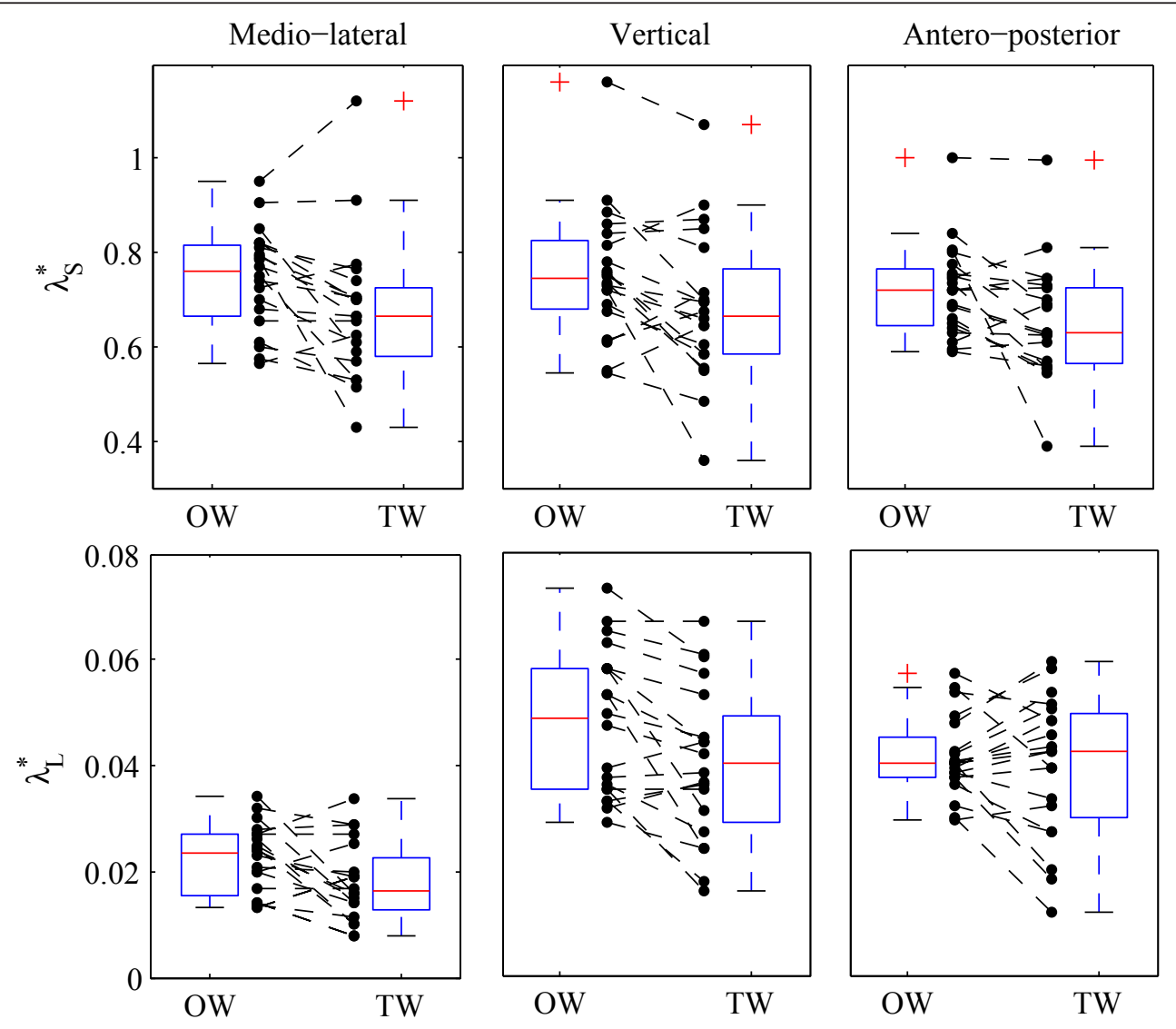

Figure 5 Individual changes of dynamic stability $\left(\lambda^{*}\right)$. Lyapunov exponent $\lambda_{L}{ }^{*}$ and $\lambda_{S}{ }^{*}$ of the 20 subjects are presented for Overground Walking (OW) and Treadmill Walking (TW). Discontinuous lines join OW and TW results. Boxplots show the quartiles and the median. 
Table 2 Correlation matrix

\begin{tabular}{|c|c|c|c|c|c|c|c|c|c|c|c|c|c|c|c|c|c|c|c|}
\hline \multicolumn{10}{|c|}{$\begin{array}{l}\text { Overground Walking } \\
\text { Correlation coefficients: }\end{array}$} & \multicolumn{10}{|c|}{$\begin{array}{c}\text { Treadmill Walking } \\
\text { Correlation coefficients: }\end{array}$} \\
\hline & SD ML & SD V & SD AP & $\lambda_{s}{ }^{*} \mathrm{ML}$ & $\lambda_{s}{ }^{*} \mathrm{~V}$ & $\lambda_{s}{ }^{*} \mathrm{AP}$ & $\lambda_{\mathrm{L}}{ }^{*} \mathrm{ML}$ & $\lambda_{L}{ }^{*} V$ & $\lambda_{\mathrm{L}}{ }^{*} \mathrm{AP}$ & & SD ML & SD V & SD AP & $\lambda_{s}{ }^{*} \mathrm{ML}$ & $\lambda_{\mathrm{s}}{ }^{*} \mathrm{~V}$ & $\lambda_{s}{ }^{*} \mathrm{AP}$ & $\lambda_{\mathrm{L}}{ }^{*} \mathrm{ML}$ & $\lambda_{\mathrm{L}} * \mathrm{~V}$ & $\lambda_{\mathrm{L}} * \mathrm{AP}$ \\
\hline SD ML & 1.00 & & & & & & & & & SD ML & 1.00 & & & & & & & & \\
\hline SD V & 0.95 & 1.00 & & & & & & & & SD V & 0.92 & 1.00 & & & & & & & \\
\hline SD AP & 0.14 & 0.24 & 1.00 & & & & & & & SD AP & 0.59 & 0.62 & 1.00 & & & & & & \\
\hline$\lambda_{s}{ }^{*} \mathrm{ML}$ & 0.05 & -0.04 & -0.02 & 1.00 & & & & & & $\lambda_{s}{ }^{*} \mathrm{ML}$ & 0.26 & 0.20 & -0.26 & 1.00 & & & & & \\
\hline$\lambda_{s}{ }^{*} V$ & -0.20 & -0.17 & -0.04 & 0.47 & 1.00 & & & & & $\lambda_{s}^{*} \mathrm{~V}$ & -0.17 & -0.08 & -0.50 & 0.74 & 1.00 & & & & \\
\hline$\lambda_{s}{ }^{*} \mathrm{AP}$ & -0.27 & -0.24 & -0.37 & 0.57 & 0.45 & 1.00 & & & & $\lambda_{s}{ }^{*} \mathrm{AP}$ & -0.01 & 0.08 & -0.32 & 0.77 & 0.75 & 1.00 & & & \\
\hline$\lambda_{\mathrm{L}}{ }^{*} \mathrm{ML}$ & 0.16 & 0.17 & 0.07 & -0.13 & 0.06 & -0.27 & 1.00 & & & $\lambda_{\mathrm{L}}{ }^{*} \mathrm{ML}$ & -0.51 & -0.50 & -0.56 & 0.34 & 0.41 & 0.30 & 1.00 & & \\
\hline$\lambda_{\mathrm{L}}{ }^{*} \mathrm{~V}$ & -0.31 & -0.37 & 0.10 & -0.23 & -0.24 & -0.28 & 0.41 & 1.00 & & $\lambda_{\mathrm{L}}{ }^{*} \mathrm{~V}$ & -0.76 & -0.81 & -0.47 & -0.36 & -0.14 & -0.30 & 0.53 & 1.00 & \\
\hline$\lambda_{\mathrm{L}}{ }^{*} \mathrm{AP}$ & -0.47 & -0.52 & 0.17 & -0.13 & -0.01 & -0.36 & 0.55 & 0.75 & 1.00 & $\lambda_{\mathrm{L}}{ }^{*} \mathrm{AP}$ & -0.77 & -0.82 & -0.57 & -0.37 & -0.18 & -0.21 & 0.45 & 0.86 & 1.00 \\
\hline$\alpha$ (DFA) & -0.45 & -0.35 & -0.39 & 0.09 & 0.10 & 0.51 & -0.09 & 0.08 & -0.09 & $\alpha(\mathrm{DFA})$ & -0.56 & -0.56 & -0.36 & 0.12 & 0.25 & 0.10 & 0.28 & 0.37 & 0.42 \\
\hline
\end{tabular}

Pearson's $r$ correlation coefficients between the variables. $S D=$ Mean Standard Deviation (MeanSD). $\lambda_{s}{ }^{*}=$ maximal Lyapunov exponent, short term dynamic stability. $\lambda_{\perp}{ }^{*}=$ maximal Lyapunov exponent, long term dynamic stability. $\alpha=$ scaling exponent (Detrended Fluctuation Analysis), fractal dynamics. ML, V and AP stand for respectively Medio-Lateral, Vertical and Antero-posterior. Significant correlation are bold printed $(p<0.05)$. 
Table 3 Canonical Correlation Analysis (CCA)

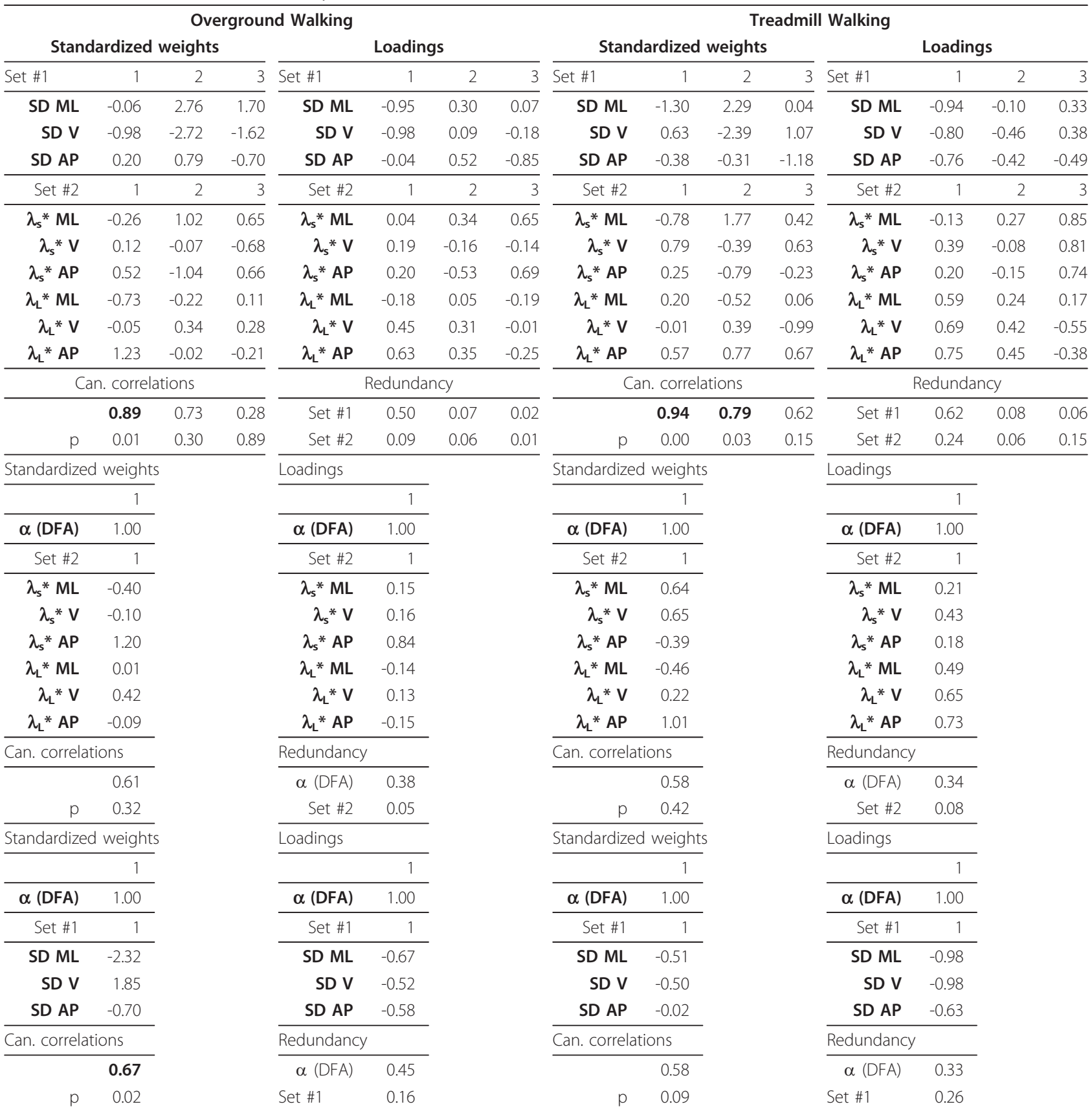

Canonical correlation analysis between 6 sets of variables. SD $=$ Mean Standard Deviation. $\lambda_{\mathrm{s}}{ }^{*}=$ maximal Lyapunov exponent, short term dynamic stability. $\lambda_{:}{ }^{*}=$ maximal Lyapunov exponent, long term dynamic stability. $\alpha=$ scaling exponent (Detrended Fluctuation Analysis), fractal dynamics. ML, $\mathrm{V}$ and AP stand for respectively Medio-Lateral, Vertical and Antero-posterior.

respective linear composites; redundancy expresses the amount of variance in one set explained by a linear composite of the other set.

\section{Results}

Treadmill effect

As presented in table 1, TW did not modify the stride-tostride kinematic variability of normalized acceleration pattern, either considering multivariate $\mathrm{T}^{2}$ statistics $(\mathrm{p}=$ 0.87 ) or individual results for each direction. TW was on average performed at slightly lower cadence than Overground Walking (OW, 3\% relative difference). The variability of stride interval was similar under both conditions. DFA of stride intervals revealed that TW changed the fractal dynamics of walking ( $-11 \%$ relative difference). Globally, multivariate analysis showed that the data are 
compatible with the assumption that TW modified dynamic stability of the gait $\left(T^{2}(6,20) p=0.0002\right)$. Five from six particular $\lambda^{*}$ exponents exhibited significant differences.

Figure 4 shows the accuracy of the effect size estimation. Non-linear estimators of gait variability $\left(\alpha, \lambda^{*}\right)$ exhibit mostly medium effect size.

Figure 5 shows the individual results of the local dynamic stability $\left(\lambda^{*}\right)$. Stability was clearly increased (lower $\lambda^{*}$ ) for a majority of subjects except for longrange Antero-Posterior stability $\lambda_{\mathrm{L}}{ }^{*}$.

Figure 6 presents the individual results of surrogate testing of fractal dynamics. The response to TW was not homogenous among subjects. Four subjects $(20 \%)$ exhibited a significant turn of long range correlations to uncorrelated pattern. For ten more subjects (50\%), a reduction was observed (more than 0.05), but outside the significant limits.

\section{Correlations}

Table 2 shows the correlation matrix (Perason's r) of the variables under both conditions. It can be observed that correlations exist between the same variables measured along different axes (for instance MeanSD ML vs. MeanSD V, $r=0.92$ ), what makes difficult the global interpretation of potential correlation among the different variability indexes.

In table 3, the results of 6 CCA are shown in details in order to explore global correlation hypotheses. The data seem compatible with the hypothesis that a negative correlation exists between kinematic variability (MeanSD) and local dynamic stability $\left(\lambda^{*}\right)$ under TW condition. Namely, two significant canonical roots $\left(R^{2}=\right.$ 0.88 and 0.62 ) indicates that the canonical variates share an important variance. In addition, the canonical loadings show that the canonical model extract a substantial portion of the variance from the variables $(70 \%$ from the set\#1 and 27\% from the set\#2). Finally, the redundancy analysis reveals that at least $70 \%$ of the variance of the set\#2 (stability) can be explained by the set\#1 (kinematic variability). The five other CCA did not produce clear evidence for significant relationship between the analyzed sets of variables. Three CCA showed low and non significant canonical roots. Two CCA exhibited barely

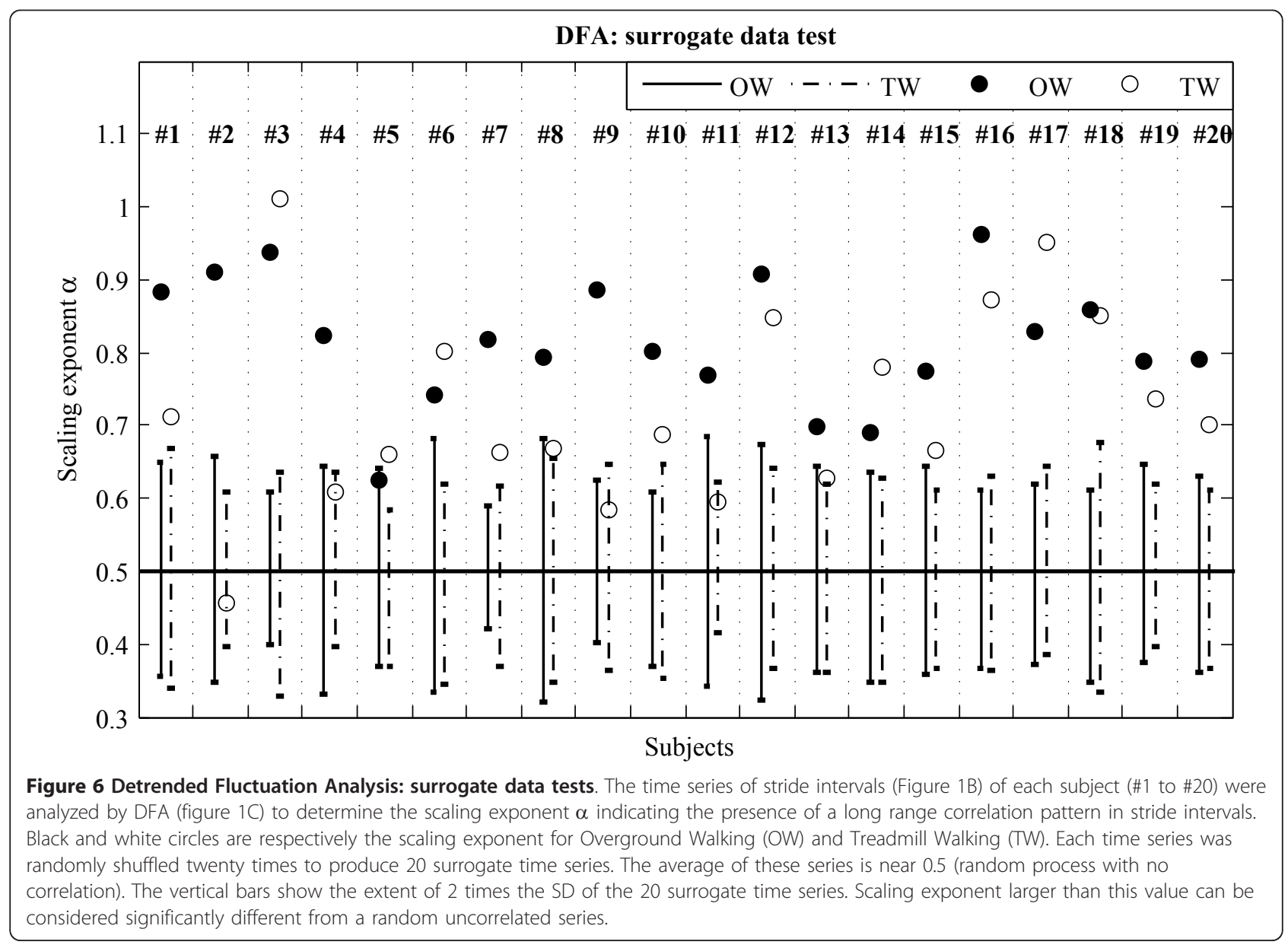


significant correlation, but the analysis of loadings showed that the canonical model did not explain a large part of the variances in the sets.

\section{Discussion}

The purpose of the present study was to analyze three gait variability indexes under two walking conditions in order to highlight modifications induced by motorized treadmill and to analyze the relationship between the indexes.

According to the working hypothesis, the results are summarized as follows:

1) As compared to Overground Walking (OW), Treadmill Walking (TW) significantly reduced the average scaling exponent (lower $\alpha$ ), but did not reverse the correlated pattern to a random or antipersistent pattern in a majority of subjects.

2) TW significantly increased local dynamic stability (lower $\lambda^{*}$ ).

3) TW did not significantly modify the kinematic variability (MeanSD).

4) No evident relationship was observed between variability indexes during OW at preferred walking speed, but in TW significant negative correlation was found between kinematic variability (MeanSD) and stability $\left(\lambda^{*}\right)$.

Overall, Conventional variability analysis (MeanSD) failed to report differences between OW and TW, whereas non-linear approaches were able to show significant changes. The variability indexes were poorly correlated together (with one exception), which might signify that each index was related to a different aspect of motor control.

\section{Technical issues}

For the present study, portable trunk accelerometry was chosen because it offers the possibility to record longterm free walking. Hence, the results concern the gait stability measured from accelerations of the low-back. Comparisons with other results should take into account that that the different gait stability studies use different kinematic variables (acceleration [7,10], positions [44], angle [8]) and different body location (thorax, head, knee, and ankle) to assess $\lambda^{*}$. We found $\lambda^{*}$ similar to those measured by others $[8,32]$, suggesting that the results are rather independent on the measurements methods.

In fractal dynamics studies, the first step is the detection of the periodic pattern of the gait in order to compute time series of stride intervals. Several methodologies have been used to measure long-term time series of stride intervals, such as foot switches [3,5], goniometer [45], video analysis [46], or high accuracy GPS [1].
Because the same variable is used (i.e. time duration of the gait cycle) for DFA analyses, data from different studies are probably comparable.

In order to increase the likelihood to point out significant correlations among variability indexes, we designed the experiment to obtain a substantial degree of standardization: we imposed the same speed $(1.25 \mathrm{~m} / \mathrm{s}, 4.5 \mathrm{~km} /$ h) for all subjects on the treadmill. This speed was chosen on the basis of a previous experiment (partially published yet [34]), which showed that the preferred speed in the same experimental conditions (same room, same treadmill) was $1.26 \pm 0.13 \mathrm{~m} / \mathrm{s}(\mathrm{n}=88)$. Similar values are found in the literature: $1.25 \mathrm{~m} / \mathrm{s}(\mathrm{n}=8)$ [47], 1.19 $\mathrm{m} / \mathrm{s}(\mathrm{n}=26)$ [48].

Walking speed was not standardized between TW and OW, as in other studies [32]. However, by selecting treadmill speed at the same speed of overground preferred speed, the results would be that subjects walk at higher speed than their preferred speed on the treadmill. Several studies showed a substantial difference between both conditions: Dal et al. [48] demonstrated that preferred walking speed determined on a treadmill is slower than overground (21\% relative difference); Marsh et al. [49] showed that, when older adults were allowed to choose a preferred walking pace, they walked faster (+61\%), used longer strides, and had a faster rate walking overground than when they walked on a treadmill. As a result, speed normalization could introduce unwanted bias. Our experimental design was therefore a compromise, which standardized speed among subjects in TW condition, but also which selected walking speed close to preferred speed, making both OW and TW conditions comparable.

In addition, Indirect clues seem to indicate that $\mathrm{TW}$ and OW conditions were quite similar: 1) stride time (which is related to walking speed) were close (3\% difference, small effect size), 2) stride time variability (CV) was the same (no significant differences), 3) no correlation was observed between stride time and other parameters (results not shown),

\section{Differences between treadmill and overground walking}

Kinematic variability, fractal dynamics (DFA) and local dynamic stability (Lyapunov exponents) quantify different aspects of locomotor control [32]. Kinematic variability describes the range in which the locomotor system operates. DFA quantify temporal dynamics of discrete events (i.e stride interval) over hundreds of consecutives strides; it assesses the presence of long-range correlations between strides, and hence analyzes the characteristics of feedbacks in locomotor control. Lyapunov exponents quantify the temporal dynamics in continuous time based on the theory of deterministic chaos; it evaluates the degree of divergence in the signal, and 
hence the resilience of the locomotor system to small perturbations. Therefore, it can be expected that these variability indexes did not react in the same way under various conditions.

These assumptions were experimentally verified in various studies that observed changes of $\lambda^{*}$ and kinematic variability between different experimental conditions or between different populations. For instance it was observed that patients with peripheral neuropathy present altered dynamic stability but normal kinematic variability $[33,50]$. Other investigators have shown that an exercise training intervention in elderly people could improve dynamic stability but not decrease kinematic variability [19].

Despite differences in the method of measurement (low-back vs. thorax acceleration) and in the experimental design (speed normalization), our results are generally in accordance with the results of Dingwell et al [32]. They analyzed only 10 healthy individuals, therefore statistical significance for small effects was more difficult to reach than in the present study. They showed a significant treadmill effect in short-term stability (lower $\left.\lambda_{S}{ }^{*}\right)$. A slight but not significant effect for long-term vertical stability (lower $\lambda_{\mathrm{L}}{ }^{*}$ ) was found. They observed that kinematic variability (MeanSD) for upper body accelerations was generally greater for OW than TW, but this trend was only significant for antero-posterior accelerations. They explained that underlying causes of differences between TW and OW were unclear: on one hand, the motorized treadmill imposed a constant nominal speed on the subjects and constrained them to walk along a much narrower and straighter path than during OW; but on the other hand, differences may have been induced by intra-stride fluctuations in treadmill belt speed, differences in mechanical compliance between the walking surfaces, and changes in visual and vestibular perceptual information. In light of the results of the present study, we hypothesize that motor control is able to maintain the same range of kinematic variability in both TW and OW conditions (same kinematic variability), probably because of compensating effects: in TW, destabilizing factors (intra-stride belt speed fluctuations, disturbing mechanical compliance, alteration of perceptual information) are balanced by stabilizing factors (constant speed, narrow and straight path). Conversely, motor control strategy adapting the gait to TW seems to specifically alter non-linear dependencies among consecutive strides: the stabilizing factors override the destabilizing ones.

In a subsequent study, Dingwell \& Marin [51] analyzed speed effect on dynamical stability $\left(\lambda_{S}{ }^{*}\right.$ and $\left.\lambda_{L}{ }^{*}\right)$ and kinematic variability (MeanSD). Walking speed was normalized by individual PWS on a treadmill. Speed range was $0.6 \mathrm{PW}$ to $1.4 \mathrm{PWS}$ by steps of 0.2 . They found significant speed effect for both $\lambda^{*}$ and MeanSD: however the effect was small for 0.8-1.2 PWS. Under our experimental conditions [34], we observed that interindivudual variability of PWS on the treadmill was low: 90\% of individuals walked in the range of 0.87-1.13 mean PWS. As a result, the speed effect among individuals in the present study was probably low. This is also indirectly confirmed by the low inter-individual variability of stride duration $(\mathrm{CV}=6 \%)$.

Fractal dynamics of stride intervals has been extensively studied by Hausdorff et al. [36]. Them and other $[1,3,52]$ have observed that constrained walking (paced cadence with a metronome), deeply modified the scaling exponent. By analogy, because treadmill also constraints the gait by imposing a constant speed, a similar effect could be expected. The results of the present study showed, in a majority of subjects, a lowering of scaling exponent to a less correlated pattern. The effect was not as strong as with paced walking [1]. The explanation could be that treadmill constrained walking speed, while metronome constrained walking pace; it could be hypothesized that the adaptation of locomotor control to external cues specifically modify correlation pattern of the constrained walking parameter, as suggested by the results of Terrier et al. [1], but this remains to be investigated.

\section{Correlations between variability indicators}

While fractal dynamics, local dynamic stability and kinematic variability characterize different features of gait variability, it is not excluded that relationships exists between them.

Jordan et al. [46] recently analyzed fractal dynamics and stability in walking/running transition on treadmill. They observed a positive correlation between $\lambda_{\mathrm{L}}{ }^{*}$ and $\alpha$ $\left(\mathrm{r}^{2}=0.65, \mathrm{~N}=12\right)$. They also observed that scaling exponent is minimal close to PWS [53] and suggested that "reduced strength of long range correlations at preferred locomotion speeds is reflective of enhanced stability and adaptability at theses speeds". Our results, using CCA, did not confirm this suggestion. No evident correlation between scaling exponent and dynamic stability was found. Several differences in the measurement method (trunk accelerometry vs 3D video analysis) and in the experimental design (high speed vs. moderate speed) may explain this divergence.

Previous studies have analyzed the relationships between variability (meanSD) and local dynamic stability $\left(\lambda_{S}{ }^{*}\right.$ and $\left.\lambda_{L}{ }^{*}\right)$. Dingwell et al. pointed out "the general lack of correlation between the standard deviation and $\lambda$ * exponents" [32]. In contrast, other investigators recently observed significant positive correlation between $\lambda_{S}{ }^{*}$ and MeanSD [54]. The results of the present study showed a counterintuitive negative 
correlation between $\lambda^{*}$ and MeanSD: during treadmill walking (but not in OW), higher kinematic variability seemed to be related to higher local stability (i.e. low $\left.\lambda^{*}\right)$. As explained above, the use of different methodologies is a potential source of divergence between studies concerning dynamic stability. It is not excluded that a confounding factor, not measured yet, related to both MeanSD and $\lambda^{*}$ could indirectly explain this correlation. Further investigations are needed to better understand the relationship between these two variability indexes.

\section{Conclusions}

Scaling exponent $(\alpha)$ and maximal Lypunov exponent $(\lambda *)$ have been advocated as a relevant indicator of neuromuscular control of stability during human locomotion $[8,32,36,55]$. The results of the present study showed that treadmill modified fractal dynamics $(\alpha)$ and local dynamic stability $(\lambda *)$ of the gait, but not kinematic variability (MeanSD). This should be kept in mind when using motorized treadmill either for fundamental research or in locomotor therapies.

Whereas both scaling exponent $(\alpha)$ and maximal Lypunov exponent $\left(\lambda^{*}\right)$ are sensitive enough to identify differences between OW and TW, they seem not correlated together. This suggests that both indexes deserve to be used in conjunction when analyzing long term gait variability, because they describe different locomotor characteristics.

\section{Acknowledgements}

The authors thank M. Antoine Bonvin for assistance in collecting experimental data. The study was supported by the Swiss accident insurance company SUVA, which is an independent, non-profit company under public law. The Intitut de Recherche en Réadaptation is supported by the State of Valais and the City of Sion.

\section{Author details}

${ }^{1}$ IRR, Institut de Recherche en Réadaptation, Sion, Switzerland. ${ }^{2}$ Clinique Romande de Réadaptation SuvaCare, Sion, Switzerland.

\section{Authors' contributions}

PT performed measurements and data analysis, and drafted the manuscript OD participated in the design and coordination of the study and assisted with drafting the manuscript. All authors read and approved the final manuscript.

\section{Competing interests}

The authors declare that they have no competing interests.

Received: 26 April 2010 Accepted: 24 February 2011

Published: 24 February 2011

\section{References}

1. Terrier $P$, Turner $V$, Schutz Y: GPS analysis of human locomotion: further evidence for long-range correlations in stride-to-stride fluctuations of gait parameters. Hum Mov Sci 2005, 24:97-115.

2. Peng CK, Buldyrev SV, Goldberger AL, Havlin S, Simons M, Stanley HE: Finite-size effects on long-range correlations: implications for analyzing DNA sequences. Phys Rev E Stat Phys Plasmas Fluids Relat Interdiscip Topics 1993, 47:3730-3733.
3. Hausdorff JM, Purdon PL, Peng CK, Ladin Z, Wei JY, Goldberger AL: Fractal dynamics of human gait: stability of long-range correlations in stride interval fluctuations. J App/ Physio/ 1996, 80:1448-1457.

4. Hausdorff JM, Peng CK, Ladin Z, Wei JY, Goldberger AL: Is walking a random walk? Evidence for long-range correlations in stride interval of human gait. J Appl Physiol 1995, 78:349-358

5. Hausdorff JM, Ashkenazy Y, Peng CK, Ivanov PC, Stanley HE, Goldberger AL: When human walking becomes random walking: fractal analysis and modeling of gait rhythm fluctuations. Physica A 2001, 302:138-147.

6. Torre K, Wagenmakers EJ: Theories and models for $1 / \mathrm{f}($ beta) noise in human movement science. Hum Mov Sci 2009, 28:297-318.

7. Rosenstein M, Collins JJ, DeLuca CJ: A practical method for calculating largest lyapunov exponents from small data sets. Physica D: Nonlinear Phenomena 1993, 65:117-134

8. Dingwell JB, Cusumano JP: Nonlinear time series analysis of normal and pathological human walking. Chaos 2000, 10:848-863.

9. Maraun D, Rust HW, Timmer J: Tempting long-memory - on the interpretation of DFA results. Nonlinear processes in Geophysics 2004 11:495-503.

10. Gates DH, Su JL, Dingwell JB: Possible Biomechanical Origins of the LongRange Correlations in Stride Intervals of Walking. Physica A 2007, 380:259-270.

11. West BJ, Latka M: Fractional Langevin model of gait variability. J Neuroeng Rehabil 2005, 2:24

12. Muñoz-Diosdado A: A non linear analysis of human gait time series based on multifractal analysis and cross correlations. J Phys: Conf Ser 2005, 23:87.

13. West BJ, Scafetta N: Nonlinear dynamical model of human gait. Phys Rev $E$ Stat Nonlin Soft Matter Phys 2003, 67:051917.

14. Bruijn SM, van Dieen JH, Meijer OG, Beek PJ: Statistical precision and sensitivity of measures of dynamic gait stability. J Neurosci Methods 2009, 178:327-333

15. Kang HG, Dingwell JB: Intra-session reliability of local dynamic stability of walking. Gait Posture 2006, 24:386-390.

16. Hausdorff JM, Cudkowicz ME, Firtion R, Wei JY, Goldberger AL: Gait variability and basal ganglia disorders: stride-to-stride variations of gait cycle timing in Parkinson's disease and Huntington's disease. Mov Disord 1998, 13:428-437

17. Khandoker AH, Taylor SB, Karmakar CK, Begg RK, Palaniswami M: Investigating scale invariant dynamics in minimum toe clearance variability of the young and elderly during treadmill walking. IEEE Trans Neural Syst Rehabil Eng 2008, 16:380-389.

18. Herman T, Giladi N, Gurevich T, Hausdorff JM: Gait instability and fractal dynamics of older adults with a "cautious" gait: why do certain older adults walk fearfully? Gait Posture 2005, 21:178-185.

19. Ohtaki Y, Arif M, Akihiro S, Fujita K, Inooka H, Nagatomi R, Tsuji I: Assessment of walking stability of elderly by means of nonlinear timeseries analysis and simple accelerometry. JSME International Journal Series C 2005, 48:607-612.

20. Hesse S: Locomotor therapy in neurorehabilitation. NeuroRehabilitation 2001, 16:133-139.

21. Hicks $A L$, Ginis $K A$ : Treadmill training after spinal cord injury: it's not just about the walking. J Rehabil Res Dev 2008, 45:241-248.

22. van Ingen Schenau GJ: Some fundamental aspects of the biomechanics of overground versus treadmill locomotion. Med Sci Sports Exerc 1980, 12:257-261.

23. Strathy GM, Chao EY, Laughman RK: Changes in knee function associated with treadmill ambulation. J Biomech 1983, 16:517-522.

24. Isacson J, Gransberg L, Knutsson E: Three-dimensional electrogoniometric gait recording. J Biomech 1986, 19:627-635.

25. White SC, Yack HJ, Tucker CA, Lin HY: Comparison of vertical ground reaction forces during overground and treadmill walking. Med Sci Sports Exerc 1998, 30:1537-1542

26. Pearce ME, Cunningham DA, Donner AP, Rechnitzer PA, Fullerton GM, Howard JH: Energy cost of treadmill and floor walking at self-selected paces. Eur J Appl Physiol Occup Physiol 1983, 52:115-119.

27. Arsenault $A B$, Winter DA, Marteniuk RG: Treadmill versus walkway locomotion in humans: an EMG study. Ergonomics 1986, 29:665-676.

28. Riley PO, Paolini G, Della Croce U, Paylo KW, Kerrigan DC: A kinematic and kinetic comparison of overground and treadmill walking in healthy subjects. Gait Posture 2007, 26:17-24. 
29. Lee SJ, Hidler J: Biomechanics of overground vs. treadmill walking in healthy individuals. J Appl Physiol 2008, 104:747-755.

30. Bruggeman H, Zosh W, Warren WH: Optic flow drives human visuolocomotor adaptation. Curr Biol 2007, 17:2035-2040.

31. Warren WH Jr, Kay BA, Zosh WD, Duchon AP, Sahuc S: Optic flow is used to control human walking. Nat Neurosci 2001, 4:213-216.

32. Dingwell JB, Cusumano JP, Cavanagh PR, Sternad D: Local dynamic stability versus kinematic variability of continuous overground and treadmill walking. J Biomech Eng 2001, 123:27-32.

33. Dingwell JB, Cusumano JP, Sternad D, Cavanagh PR: Slower speeds in patients with diabetic neuropathy lead to improved local dynamic stability of continuous overground walking. J Biomech 2000, 33:1269-1277.

34. Deriaz O, Najafi B, Ballabeni P, Crettenand A, Gobelet C, Aminian K, Rizzoli R, Gremion G: Proximal tibia volumetric bone mineral density is correlated to the magnitude of local acceleration in male long-distance runners. $J$ Appl Physiol 108:852-857.

35. Aminian K, Rezakhanlou K, De Andres E, Fritsch C, Leyvraz PF, Robert P. Temporal feature estimation during walking using miniature accelerometers: an analysis of gait improvement after hip arthroplasty. Med Biol Eng Comput 1999, 37:686-691.

36. Hausdorff JM: Gait dynamics, fractals and falls: finding meaning in the stride-to-stride fluctuations of human walking. Hum Mov Sci 2007, 26:555-589.

37. Hausdorff JM, Mitchell SL, Firtion R, Peng CK, Cudkowicz ME, Wei JY, Goldberger AL: Altered fractal dynamics of gait: reduced stride-interval correlations with aging and Huntington's disease. J Appl Physiol 1997, 82:262-269.

38. Takens F: Detecting strange attractors in turbulence. In Dynamical Systems and Turbulence. Edited by: Rands D, Young LS. Berlin, Heidelberg, New-York: Springer; 1980:366-381.

39. Fraser $\mathrm{AM}$, Swinney $\mathrm{HL}$ : Independent coordinates for strange attractors from mutual information. Phys Rev A 1986, 33:1134-1140.

40. Kennel MB, Brown R, Abarbanel HD: Determining embedding dimension for phase-space reconstruction using a geometrical construction. Phys Rev A 1992, 45:3403-3411.

41. A practical method for calculating largest lyapunov exponents from small data sets. [http://www.physionet.org/physiotools/lyapunov/11d2/].

42. Nakagawa S, Cuthill IC: Effect size, confidence interval and statistical significance: a practical guide for biologists. Biol Rev Camb Philos Soc 2007, 82:591-605.

43. Hair JF, Anderson RE, Tatham RL: Multivariate data analysis with readings. 2 edition. New York, London: Macmillan; 1987.

44. Moraiti C, Stergiou N, Ristanis S, Georgoulis AD: ACL deficiency affects stride-to-stride variability as measured using nonlinear methodology. Knee Surg Sports Traumatol Arthrosc 2007, 15:1406-1413.

45. Gates DH, Dingwell JB: Peripheral neuropathy does not alter the fractal dynamics of stride intervals of gait. J Appl Physiol 2007, 102:965-971.

46. Jordan $\mathrm{K}$, Challis JH, Cusumano JP, Newell KM: Stability and the timedependent structure of gait variability in walking and running. Hum Mov Sci 2009, 28:113-128.

47. Holt KJ, Jeng SF, Rr RR, Hamill J: Energetic Cost and Stability During Human Walking at the Preferred Stride Velocity. J Mot Behav 1995, 27:164-178.

48. Dal U, Erdogan T, Resitoglu B, Beydagi H: Determination of preferred walking speed on treadmill may lead to high oxygen cost on treadmill walking. Gait Posture 31:366-369.

49. Marsh AP, Katula JA, Pacchia CF, Johnson LC, Koury KL, Rejeski WJ: Effect of treadmill and overground walking on function and attitudes in older adults. Med Sci Sports Exerc 2006, 38:1157-1164.

50. Dingwell JB, Ulbrecht JS, Boch J, Becker MB, O'Gorman JT, Cavanagh PR: Neuropathic gait shows only trends towards increased variability of sagittal plane kinematics during treadmill locomotion. Gait Posture 1999, 10:21-29.

51. Dingwell JB, Marin LC: Kinematic variability and local dynamic stability of upper body motions when walking at different speeds. J Biomech 2006, 39:444-452.

52. Delignieres $D$, Torre K: Fractal dynamics of human gait: a reassessment of the 1996 data of Hausdorff et al. J Appl Physiol 2009, 106:1272-1279.

53. Jordan $\mathrm{K}$, Challis JH, Newell KM: Walking speed influences on gait cycle variability. Gait Posture 2007, 26:128-134.
54. Bruijn SM, van Dieen JH, Meijer OG, Beek PJ: Is slow walking more stable? J Biomech 2009, 42:1506-1512.

55. Goldberger AL, Amaral LA, Hausdorff JM, Ivanov P, Peng CK, Stanley HE: Fractal dynamics in physiology: alterations with disease and aging. Proc Natl Acad Sci USA 2002, 99(Suppl 1):2466-2472.

doi:10.1186/1743-0003-8-12

Cite this article as: Terrier and Dériaz: Kinematic variability, fractal dynamics and local dynamic stability of treadmill walking. Journal of NeuroEngineering and Rehabilitation 2011 8:12.

\section{Submit your next manuscript to BioMed Central and take full advantage of:}

- Convenient online submission

- Thorough peer review

- No space constraints or color figure charges

- Immediate publication on acceptance

- Inclusion in PubMed, CAS, Scopus and Google Scholar

- Research which is freely available for redistribution
Ciomed Central 